\title{
Respiratory Monitoring by Inductive Plethysmography in Unrestrained Subjects Using Position Sensor-Adjusted Calibration
}

\author{
Gregor Brüllmann ${ }^{\mathrm{a}}$ Karsten Fritsch ${ }^{\mathrm{a}}$ Robert Thurnheer ${ }^{\mathrm{c}}$ Konrad E. Bloch ${ }^{\mathrm{a}, \mathrm{b}}$

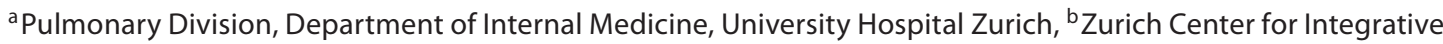 \\ Human Physiology, University of Zurich, Zurich, and 'Pulmonary Division, Cantonal Hospital Munsterlingen, \\ Scherzingen, Switzerland
}

\section{Key Words}

Inductive plethysmography · Pulmonary ventilation •

Exercise $\cdot$ Noninvasive physiologic monitoring $\cdot$ Breathing pattern

\begin{abstract}
Background: Portable respiratory inductive plethysmography (RIP) is promising for noninvasive monitoring of breathing patterns in unrestrained subjects. However, its use has been hampered by requiring recalibration after changes in body position. Objectives: To facilitate RIP application in unrestrained subjects, we developed a technique for adjustment of RIP calibration using position sensor feedback. Methods: Five healthy subjects and 12 patients with lung disease were monitored by portable RIP with sensors incorporated within a body garment. Unrestrained individuals were studied during 40-60 min while supine, sitting and upright/walking. Position was changed repeatedly every 5$10 \mathrm{~min}$. Initial qualitative diagnostic calibration followed by volume scaling in absolute units during 20 breaths in different positions by flow meter provided position-specific volume-motion coefficients for RIP. These were applied during subsequent monitoring in corresponding positions according to feedback from 4 accelerometers placed at the chest and thigh. Accuracy of RIP was evaluated by face mask pneumotachography. Results: Position sensor feedback allowed
\end{abstract}

accurate adjustment of RIP calibration during repeated position changes in subjects and patients as reflected in a minor mean difference (bias) in breath-by-breath tidal volumes estimated by RIP and flow meter of 0.02 liters (not significant) and limits of agreement ( \pm 2 SD) of $\pm 19 \%$ (2,917 comparisons). An average of 10 breaths improved precision of RIP (limits of agreement $\pm 14 \%$ ). Conclusions: RIP calibration incorporating position sensor feedback greatly enhances the application of RIP as a valuable, unobtrusive tool to investigate respiratory physiology and ventilatory limitation in unrestrained healthy subjects and patients with lung disease during everyday activities including position changes.

Copyright $\odot 2009$ S. Karger AG, Basel

\section{Introduction}

Respiratory inductive plethysmography (RIP) is a valuable tool for monitoring ventilation and breathing patterns without airway instrumentation [1]. It has wide applications, including evaluation of sleep-related breathing disorders [2-4], and respiratory monitoring in critical care and anesthesia $[5,6]$ as well as in other areas of physiologic and clinical research $[7,8]$. Unlike optometric respiratory monitoring [9], RIP is available as portable equipment and can be applied in unrestrained ambulatory subjects during daily activities $[7,10]$.

\section{KARGER}

Fax +4161306 1234 E-Mail karger@karger.ch www.karger.com
(C) 2009 S. Karger AG, Basel 0025-7931/10/0792-0112\$26.00/0

Accessible online at: www.karger.com/res
Konrad E. Bloch, MD

Pulmonary Division, Department of Internal Medicine

University Hospital of Zurich, Rämistrasse 100

CH-8091 Zurich (Switzerland)

Tel. +41 4425538 28, Fax +41 4425544 51, E-Mail konrad.bloch@usz.ch 
According to the principles of RIP, 2 degrees of freedom of chest wall motion are monitored by 2 inductance sensors at the level of the nipples and the umbilicus, respectively $[11,12]$. Calibration of RIP provides volumemotion coefficients for the 2 inductance signals so that their sum, Vsum(RIP), reflects lung volume changes. Since volume-motion coefficients for RIP sensors are altered by body position $[13,14]$, recalibration is required each time the position is changed. This has hampered ambulatory monitoring by RIP.

To overcome this drawback, the purpose of the current investigation was to develop a method for position-specific automatic calibration of RIP during changes in body position based on position sensor feedback. We hypothesized that in a given individual and position, volumemotion coefficients would remain constant. Therefore, their ratio $(\mathrm{K})$ and gain $(\mathrm{M})$ could be determined once during initial calibration for several body positions and applied during subsequent monitoring according to the feedback from body position sensors. We further hypothesized that application of position-specific volume-motion coefficients improved accuracy of RIP and allowed its application in subjects during changes in body positions. To evaluate these hypotheses, we employed a portable RIP device as described previously [10] in conjunction with 4 accelerometers ( 3 placed on the chest wall, 1 on a thigh) that determined body position and motion. Tidal volumes monitored by the portable RIP device incorporating body position feedback were compared to corresponding values obtained by a flow meter attached to a face mask in ambulatory healthy subjects and in patients with lung disease. The results of our study confirm that the novel concept of position sensor-adjusted calibration of RIP greatly enhances the applications of noninvasive respiratory monitoring by providing the opportunity to study ventilatory effects of pulmonary and cardiac disease and their treatment, or to document effects of environmental conditions in unrestrained subjects and patients during activities of daily life.

\section{Methods}

\section{Subjects and Patients}

Five healthy volunteers (age 23-26 years), 10 patients with chronic obstructive pulmonary disease (mean age \pm SD $49 \pm 21$ years; mean $\mathrm{FEV}_{1} \pm$ SD $2.15 \pm 1.17$ liters, $53 \pm 22 \%$ predicted) and 2 patients with restrictive lung disease [age 49 and 57 years, FVC 1.92 liters (48\% predicted) and 3.24 liters (79\% predicted)] gave informed consent to participate in the study, which was approved by the hospital ethics committee.
Measurements

RIP was performed with a portable device (LifeShirt ${ }^{\mathrm{TM}}$; VivoMetrics, Ventura, Calif., USA) as described previously [10]. Sensors were sewn in a sinusoidal array into a sleeveless elastic shirt so that they encircled the rib cage and abdomen at the level of the nipples and the umbilicus. To prevent the lower end of the shirt from moving upward during movements, it was fixed to the trousers of the individuals by safety pins. Three accelerometers for measurement of body movements and position in 3 axes (spinal, transverse and frontal) were incorporated in the shirt, and $1 \mathrm{ac}-$ celerometer was attached to 1 thigh to differentiate sitting from standing positions and detect leg movements (fig. 1). RIP and accelerometer signals, a 3-lead ECG and the signal of a portable flow meter (Spiroson; Isler Engineering, Dürnten, Switzerland) attached to a face mask were recorded by a portable, battery-powered recording unit (the LifeShirt monitor weighed $382 \mathrm{~g}$ ) that was worn in a bag. Signals were sampled at $50 \mathrm{~Hz}$ with 12-bit resolution.

\section{Protocol}

At the beginning of measurements, the flow meter was calibrated with a 1-liter syringe and then attached to the face mask. The face mask was fitted and tested to be airtight during occlusion of the flow meter. The healthy volunteers were monitored during approximately $60 \mathrm{~min}$ while resting supine and while standing, each during approximately $10 \mathrm{~min}$ at a time with a total of 5 position changes in random order. Patients were monitored for approximately $90 \mathrm{~min}$ while sitting, supine and walking, each for approximately $10 \mathrm{~min}$ at a time, with a total of 8 body position changes in random order.

\section{Data Analysis and Statistics}

Data were downloaded from the recording unit and analyzed by dedicated software that provided time series of raw signals and breath-by-breath breathing pattern variables (VivoLogic ${ }^{\mathrm{TM}}$; VivoMetrics, Ventura, Calif., USA) [6, 10, 15].

The qualitative diagnostic calibration method (QDC) described by Sackner et al. [14] was used to derive the ratio of the rib cage/abdominal volume-motion coefficients $(\mathrm{K})$ from the standard deviation of compartmental motion during 5 min of stable natural breathing at a nearly constant tidal volume according to isovolume calibration principles: $\mathrm{K}=-\mathrm{SD}(\Delta \mathrm{uVAB}) / \mathrm{SD}(\Delta \mathrm{uVRC})$, where $\triangle \mathrm{uVRC}$ and $\triangle \mathrm{uVAB}$ are the uncalibrated rib cage and abdominal RIP tidal signals, respectively. Relative changes in lung volume Vsum(RIP) in arbitrary units were computed as the sum of abdominal plus rib cage volume changes: Vsum(RIP) $=\mathrm{VAB}+$ VRC. In a second step, the gain of Vsum(RIP) was calibrated in absolute volume units (liters) by deriving a scaling factor (M) from comparison of RIP-derived tidal volumes with those from simultaneous flow meter recordings during 20 breaths. Body positions (supine, sitting, standing/walking) were identified from the pattern of the 4 accelerometer signals (fig. 1,2) and corresponding $\mathrm{K}$ ratios and gain factors $(\mathrm{M})$ were determined separately for each position. As a result, 3 sets of $\mathrm{K}$ ratios and related scaling factors $M$ corresponding to supine, sitting and standing/ walking positions, respectively, were obtained and stored. They were applied to calibrate and recalibrate the RIP wave forms over the course of the monitoring period according to the position determined continuously by the accelerometers. 
Fig. 1. Schematic illustration of body position determination by accelerometers. Three are placed on the chest (in the frontal, transverse and spinal axis, respectively), 1 on the thigh (in the frontal axis). The pattern of angles relative to gravity indicated by the 4 accelerometer signals allows to distinguish the body positions standing/walking, sitting and horizontal supine (and prone/lateral decubitus). The actual size of accelerometers is so small that they are integrated in the cable connecting RIP sensors with the recording unit.

Fig. 2. Example of a recording obtained in a patient with chronic obstructive lung disease. RIP volume signals of the rib cage, the abdomen and the sum are displayed along with the signals of the flow meter and the signals of 4 accelerometers that indicate the angle between their axis and gravity. Three accelerometers are arranged within the body garment at the chest in the spinal, frontal and transverse axis, respectively, to detect upright, supine, prone and lateral decubitus positions. The 4 th accelerometer is attached to the thigh and oriented in the frontal axis to differentiate supine/sitting from standing. QDC is performed in each position to obtain the ratio of abdominal/rib cage volume-motion coefficients (K). The gain of the RIP sum volume (M) is scaled in liters by comparison to the integrated output of the flow meter in each position during 20 breaths. Calibration factors are stored and applied to subsequent recording periods in the corresponding position.
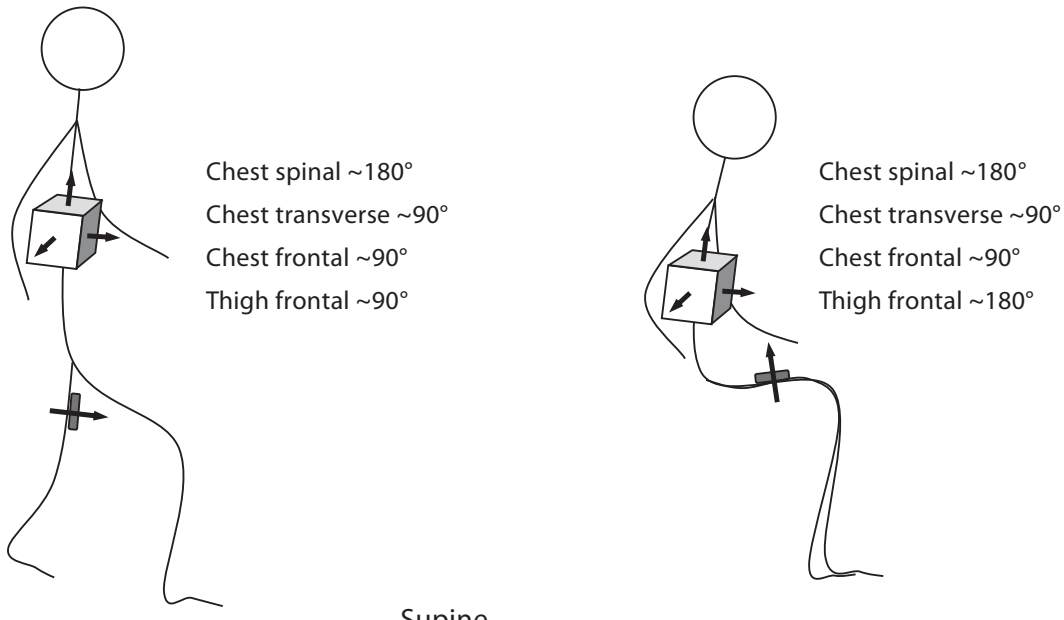

Supine

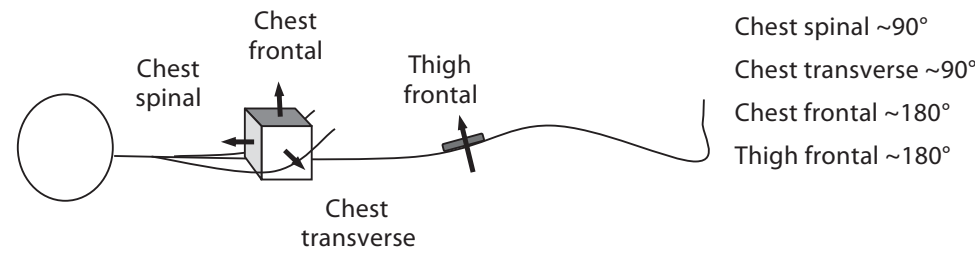

Chest spinal $\sim 90^{\circ}$

ansverse $\sim 90^{\circ}$

Chest frontal $\sim 180^{\circ}$

Chest
transverse

sitting supine walking

Rib cage

volume

Abomen volume

Sum

volume

Flow meter air flow

Spinal

axis chest

Frontal

axis chest

Transverse

axis chest

Frontal axis thigh

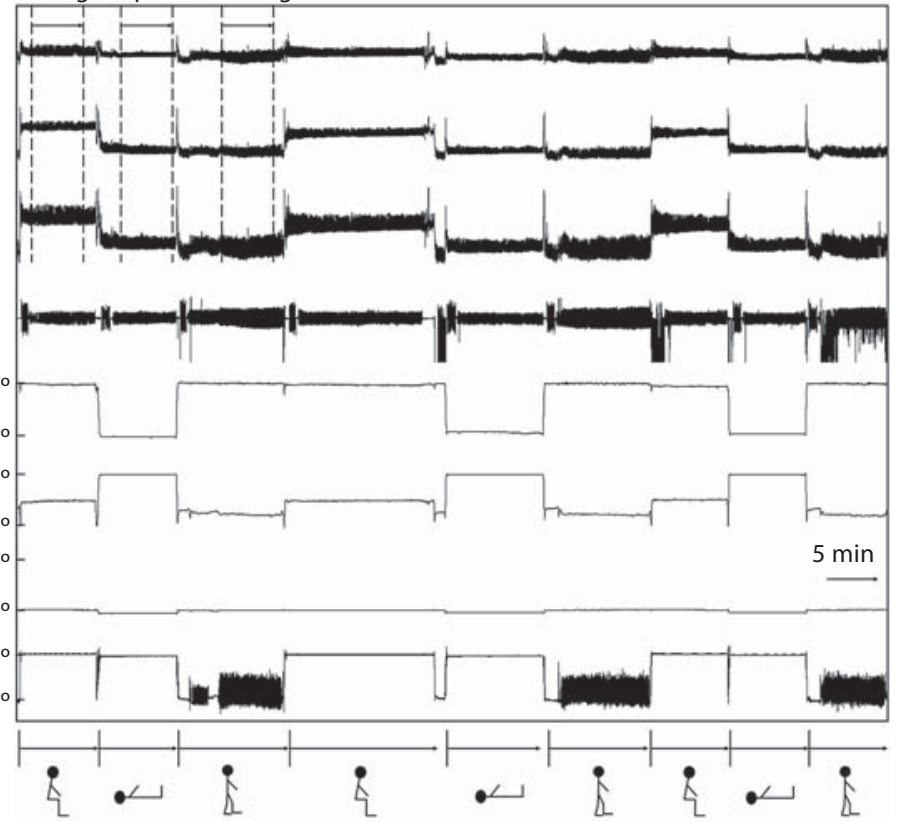



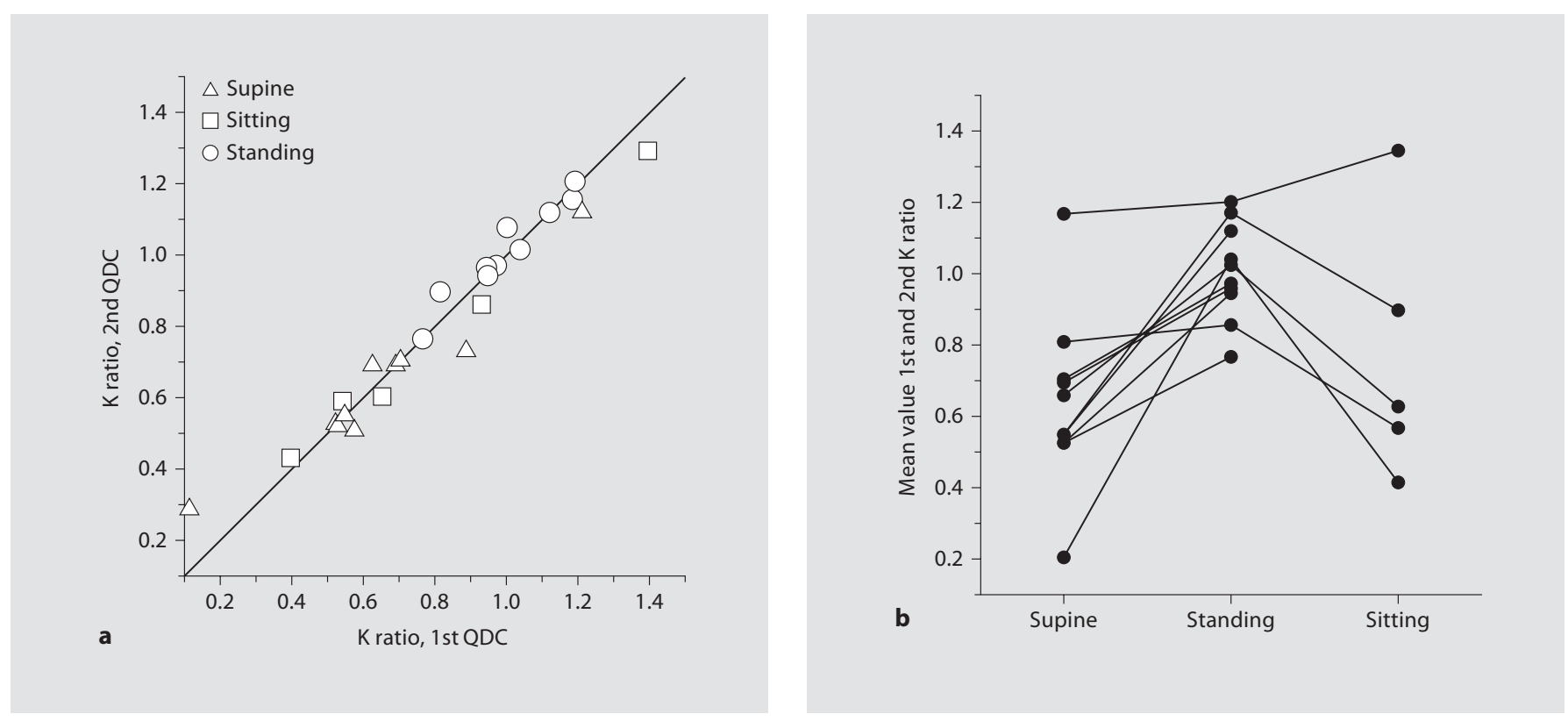

Fig. 3. In 5 healthy subjects and 5 patients, QDC was performed twice over the course of experiments with several position changes between the two QDC. a Identity plot of K ratios from the second versus the first individual QDC in the corresponding body position. There was close agreement between individual values in any particular position. $\mathbf{b}$ Changes in individual mean $\mathrm{K}$ ratios from one to another position. Healthy subjects were studied in supine and standing position, patients in supine, standing and sitting position. Within individuals, the $\mathrm{K}$ ratio was highest in the standing position with one exception.

Accuracy of RIP-derived tidal volumes in various positions was evaluated by comparison to corresponding values from the flow meter outside the calibration period in terms of the mean difference (bias) and the limits of agreement ( \pm 2 SD) [16]. Statistical significance was evaluated by $\mathrm{t}$ tests and a probability of $\mathrm{p}<$ 0.05 was considered significant. To verify whether the application of position-specific $\mathrm{K}$ ratios and $\mathrm{M}$ factors provided superior accuracy of RIP compared to the application of only one single K ratio and $\mathrm{M}$ factor obtained in supine position to all positions, agreement with the flow meter was tested separately for both analyses (with and without body position adjustment of calibration). To investigate the stability of $\mathrm{K}$ ratios after repeated changes in body positions, the $\mathrm{K}$ ratio obtained by a first QDC performed at the beginning of a recording session was compared to a second QDC performed in the corresponding position later in the course of the session after several position changes.

\section{Results}

A satisfactory, snug fit of the available standard sizes of RIP garments was achieved in all subjects and patients, and RIP recordings were successfully obtained in all of them. Figure 2 shows a representative recording in a male patient with chronic obstructive lung disease. Body posi- tions were identified from the pattern of the signals of the 3 accelerometers incorporated in the garment and the one placed at the thigh for distinction of sitting from standing/walking position.

\section{Stability of Volume-Motion Coefficients (K Ratios)}

In 5 healthy subjects and 5 patients, QDC was performed twice on different portions of the recordings in supine and standing positions, respectively, and in patients additionally in sitting position after they had changed their position several times. A graphic comparison of individual position-specific pairs of $\mathrm{K}$ ratios obtained in various positions during a first and a second QDC in the respective position revealed close agreement in both healthy subjects and patients, thereby demonstrating stability over time despite several changes in body positions (identity plot in fig. 3a). The mean difference between the first and second $K$ ratio was $0 \pm 0.04$ $(\mathrm{p}=0.96)$. In figure $3 \mathrm{~b}$, the individual mean value of the $\mathrm{K}$ factor in the different positions is displayed. The plot reveals greater $\mathrm{K}$ ratios in standing than in other positions with exception of 1 patient in whom the $\mathrm{K}$ ratio was highest while sitting (fig. $3 \mathrm{~b}$ ). 

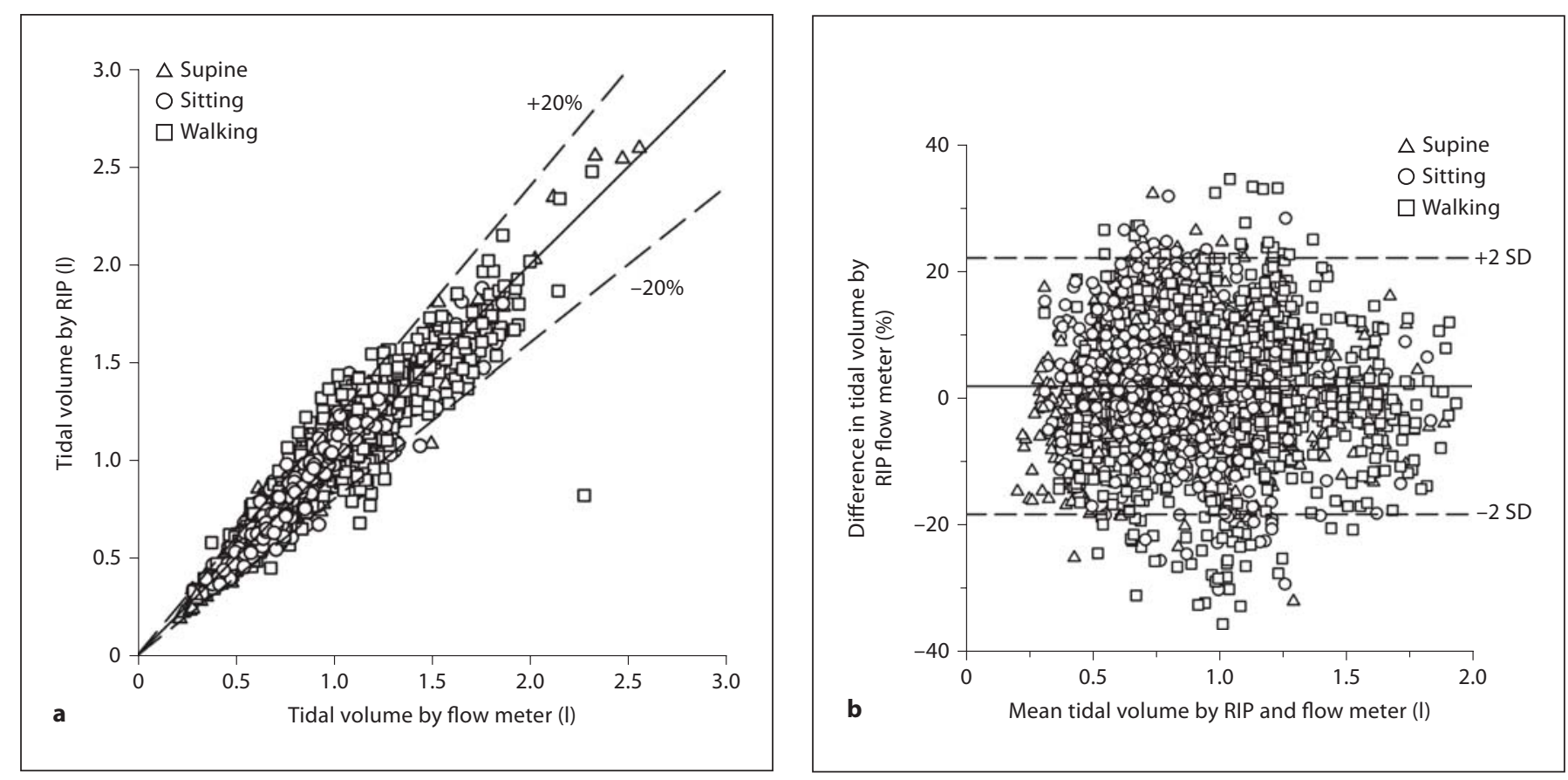

Fig. 4. a Identity plot of breath-by-breath tidal volumes recorded by RIP versus corresponding values by the flow meter in 5 healthy subjects and 10 patients. b Differences in breath-by-breath tidal volumes by RIP and flow meter are plotted against their mean values. The solid and dashed lines represent the mean difference (bias) and the limits of agreement ( \pm 2 SD of the bias), respectively.

Tidal Volumes by RIP Using Position-Specific

Calibration Based on Position Sensor Feedback

Accuracy of breath by breath tidal volumes derived by RIP using position-specific calibration was graphically and numerically evaluated by Bland-Altman analysis (fig. 4). The table summarizes the results of the breathby-breath analysis in all subjects and patients for each body position. The mean differences between RIP and flow meter-derived values (bias) were not statistically significant. The limits of agreement were improved if 10 breath averages were compared between methods (fig. 5; table 1).

\section{Accuracy of RIP with Single-Position Calibration versus Several Position-Specific Calibrations}

In 10 patients, the QDC and flow meter calibrations were performed in a single position (supine) and the derived $\mathrm{K}$ ratio and gain factor were applied to all positions. Agreement between RIP and flow meter-derived tidal volumes obtained in this way was compared to corresponding values obtained by an analysis that used corresponding position-specific sets of $\mathrm{K}$ ratios and scaling factors $M$ from calibration in supine, sitting and stand- ing/walking positions (fig. 6). Position-specific calibration provided tidal volumes with significantly greater accuracy in terms of bias (mean \pm SD value of $0.01 \pm 0.05$ liters) and limits of agreement (17 $\pm 7 \%)$ compared to the single-posture calibration (mean bias \pm SD of $0.21 \pm$ 0.48 liters; mean limits of agreement $22 \pm 14 \%, \mathrm{p}<0.05$ vs. position-specific calibration, both instances).

\section{Discussion}

We describe a novel method for position-dependent calibration of RIP based on feedback from position sensors placed at the chest and thigh. We confirmed previous findings of major alterations in RIP calibration with positional changes (fig. 3,6). We further demonstrated that the ratio of abdominal/rib cage volume-motion coefficients of RIP sensors (the K ratio) and the scaling (factor $\mathrm{M})$ of the RIP sum signal remained stable over time in a specific position and resumed the same value after transient positional changes (fig. 3). Based on these premises, the proposed method comprises initial calibration in various positions to obtain individual sets of $\mathrm{K}$ and $\mathrm{M}$ for 

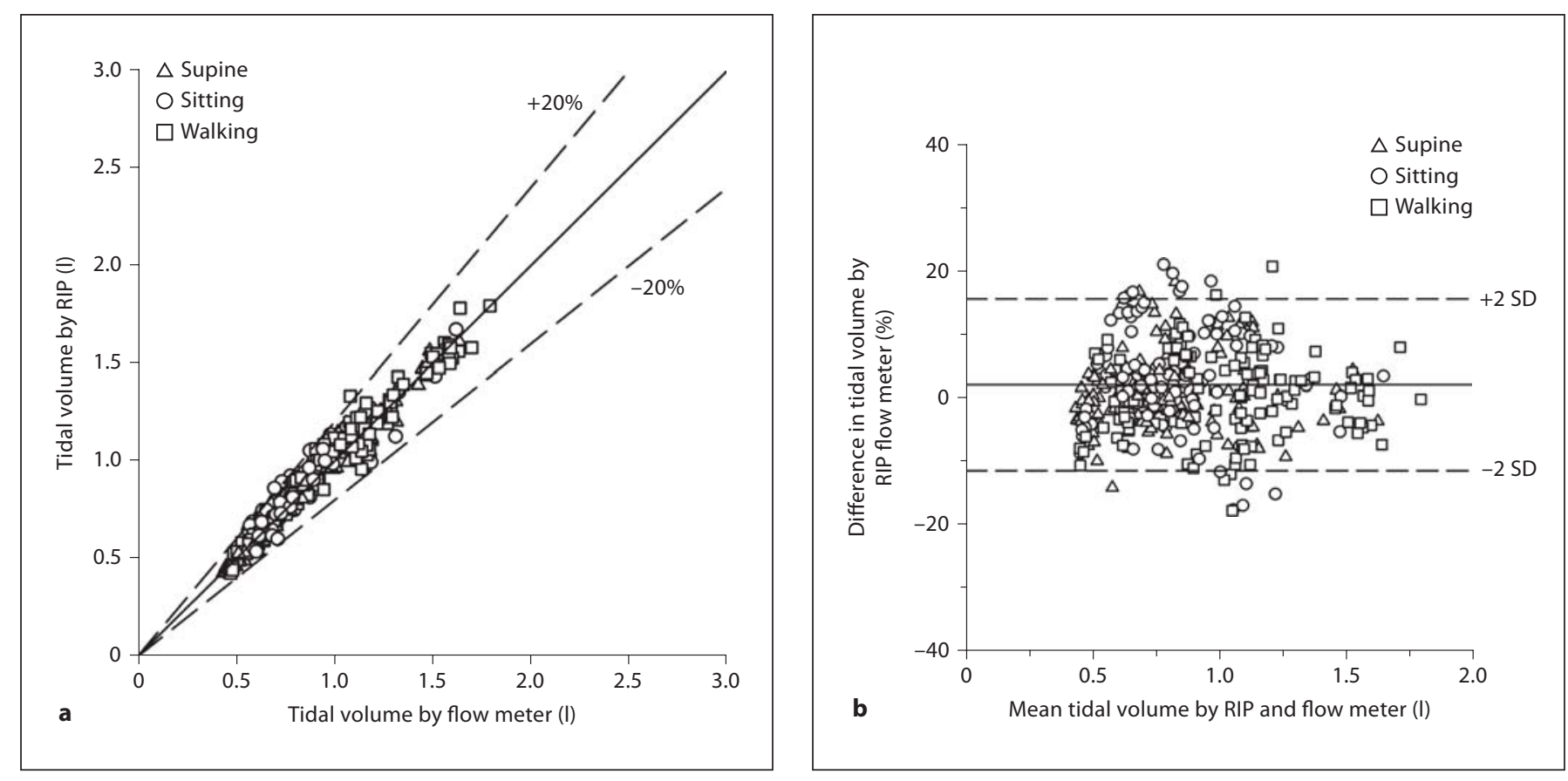

Fig. 5. a Identity plot of the mean values of 10 tidal volumes recorded by RIP versus corresponding values by the flow meter in 5 healthy subjects and 10 patients. b Differences in mean values of 10 tidal volumes recorded by RIP and flow meter are plotted against mean values from both methods. The solid and dashed lines represent the mean difference (bias) and the limits of agreement ( \pm 2 SD of the bias), respectively.

various positions that are subsequently applied to recalibrate RIP according to the actual position detected by accelerometers. Compared to face mask pneumotachography, the novel calibration technique for RIP provided accurate estimates of tidal volumes in healthy subjects and in patients with lung disease in various positions at rest and during walking (fig. 4, 5). Therefore, portable RIP incorporating position sensor feedback-controlled calibration holds promise as a noninvasive and convenient method for unobtrusive monitoring of ventilation in ambulatory, unrestrained subjects and patients during various activities.

Accurate calibration of RIP controlled by position sensors has several requirements. A crucial point is prevention of RIP sensor displacement during movements and changes in body position. This has been achieved in the current study by using a snugly fitting body garment, the LifeShirt, which holds the incorporated RIP sensors firmly in place. Additionally, the lower end of the shirt was fixed to the trousers of the individuals by pins to prevent upward slipping. Stable ratios of abdominal/rib cage volume-motion coefficients $\mathrm{K}$ over the course of experiments (fig. 3) were consistent with absent or only mini- mal sensor displacement despite multiple position changes and walking. A small-size and lightweight RIP record$\mathrm{er}$ is a further requirement for convenient monitoring of unrestrained ambulatory subjects and patients.

Our observation of considerable inaccuracies of RIPderived tidal volumes obtained in a position other than that during calibration (fig. 6) are in line with earlier findings of Zimmerman et al. [13] using the isovolume calibration [11] and the 2 positions simultaneous equations methods [17], respectively. Correspondingly, Sackner et al. [14] reported that accuracy of RIP using QDC in supine posture was reduced when the same calibration coefficients were applied to recordings obtained in sitting position. Other studies using the 2 positions, simultaneous equations and linear regression calibration methods that derive volume-motion coefficients from simultaneous spirometric and RIP recordings in recumbent and semi-recumbent or upright position have provided acceptable results in various positions [18]. However, based on the current data and the cited studies $[13,14]$ we conclude that optimal accuracy of RIP in one specific posture is obtained if a single-posture calibration method is performed in the same position as that during monitoring 

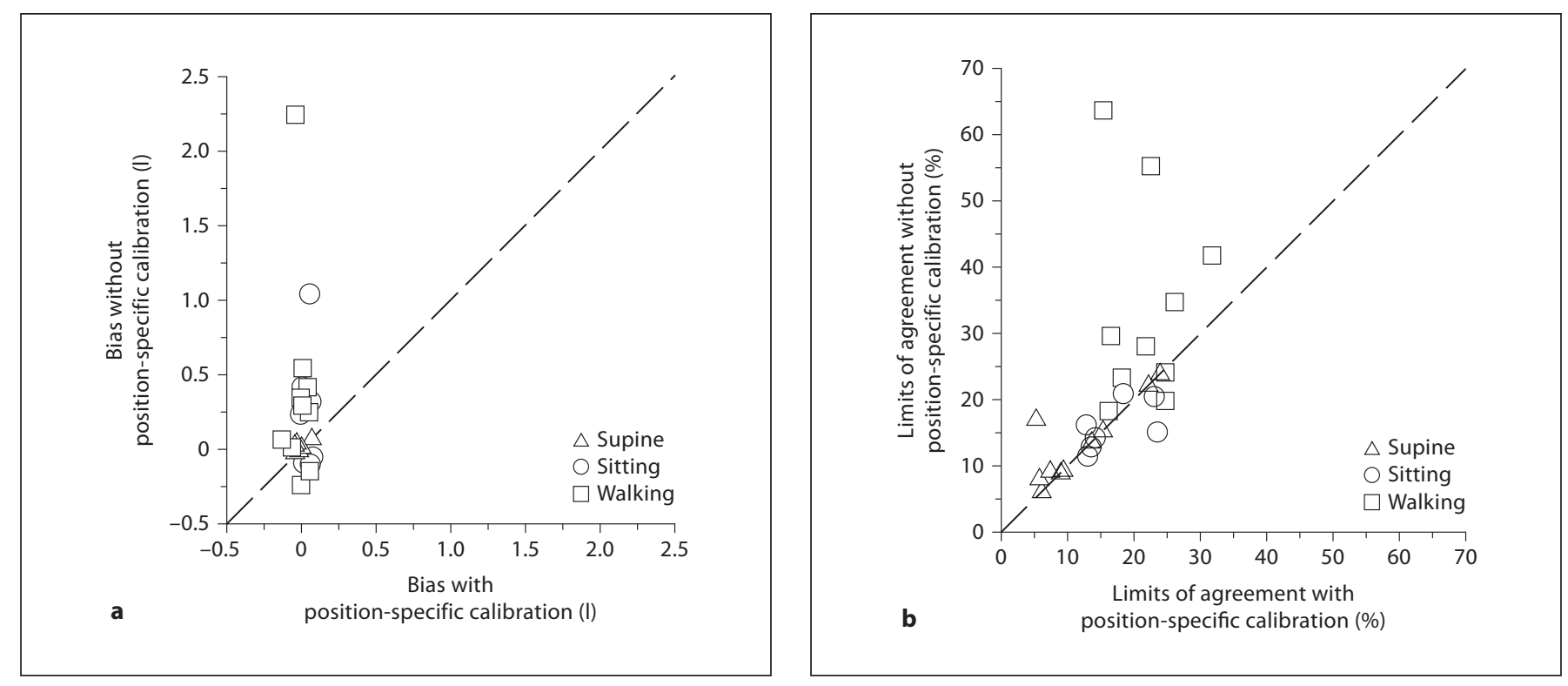

Fig. 6. In 10 patients, recordings were analyzed with position-specific QDC according to position sensor feedback and with QDC in supine position applied to all positions. a Identity plot of bias of RIP versus flow meterderived tidal volumes with QDC in supine position only versus corresponding values of bias with position-specific QDC. b Identity plot of corresponding limits of agreement. Position-specific QDC provided greater accuracy and precision of tidal volume estimates, in particular during walking.

Table 1. Accuracy of tidal volumes derived by RIP based on position-specific calibration

\begin{tabular}{|c|c|c|c|c|}
\hline & Supine & Sitting & Standing/walking & All positions \\
\hline \multicolumn{5}{|l|}{ Healthy subjects $(n=5)$} \\
\hline Breaths & 200 & NA & 200 & 400 \\
\hline Mean \pm SD tidal volume, 1 & $0.71 \pm 0.15$ & & $0.75 \pm 0.19$ & $0.73 \pm 0.18$ \\
\hline Bias, 1 & 0.00 & & 0.00 & 0.00 \\
\hline \multicolumn{5}{|l|}{ Limits of agreement } \\
\hline Breath by breath, 1 & $\pm 0.08( \pm 11 \%)$ & & $\pm 0.14( \pm 18 \%)$ & $\pm 0.11( \pm 15 \%)$ \\
\hline 10 breaths average, 1 & $\pm 0.05( \pm 7 \%)$ & & $\pm 0.11( \pm 14 \%)$ & $\pm 0.08( \pm 11 \%)$ \\
\hline \multicolumn{5}{|c|}{ Patients with lung disease $(n=12)$} \\
\hline Breaths & 930 & 737 & 850 & 2,517 \\
\hline Mean \pm SD tidal volume, 1 & $0.78 \pm 0.32$ & $0.84 \pm 0.27$ & $1.03 \pm 0.36$ & $0.88 \pm 0.34$ \\
\hline Bias, 1 & 0.01 & 0.04 & 0.01 & 0.02 \\
\hline \multicolumn{5}{|l|}{ Limits of agreement } \\
\hline Breath by breath, 1 & $\pm 0.14( \pm 14 \%)$ & $\pm 0.17( \pm 18 \%)$ & $\pm 0.26( \pm 24 \%)$ & $\pm 0.20( \pm 19 \%)$ \\
\hline 10 breath average, 1 & $\pm 0.10( \pm 12 \%)$ & $\pm 0.13( \pm 13 \%)$ & $\pm 0.14( \pm 13 \%)$ & $\pm 0.13( \pm 14 \%)$ \\
\hline \multicolumn{5}{|c|}{ Healthy subjects and patients combined $(n=17)$} \\
\hline Breaths & 1,130 & 737 & 1,050 & 2,917 \\
\hline Mean \pm SD tidal volume, 1 & $0.77 \pm 0.30$ & $0.84 \pm 0.27$ & $0.98 \pm 0.35$ & $0.86 \pm 0.33$ \\
\hline Bias, 1 & 0.01 & 0.04 & 0.01 & 0.02 \\
\hline \multicolumn{5}{|l|}{ Limits of agreement } \\
\hline Breath by breath, 1 & $\pm 0.13( \pm 14 \%)$ & $\pm 0.17( \pm 18 \%)$ & $\pm 0.24( \pm 23 \%)$ & $\pm 0.19( \pm 20 \%)$ \\
\hline 10 breaths average, 1 & $\pm 0.10( \pm 11 \%)$ & $\pm 0.13( \pm 13 \%)$ & $\pm 0.13( \pm 13 \%)$ & $\pm 0.12( \pm 14 \%)$ \\
\hline
\end{tabular}

RIP tidal volumes were compared with corresponding values derived by flow meter. Bias: mean difference between value by RIP and flow meter (no significance for bias in all comparisons). LA = Limits of agreement, that is, 2 SD of bias; NA = not applicable. 
and if RIP is calibrated in each position of interest. Consistent with a decrease in the rib cage/abdominal isovolume angle reported by Zimmerman et al. [13], with change from upright to semi-recumbent or supine position we observed greater $\mathrm{K}$ ratios in standing than in sitting or supine position. The considerable interindividual variability of change in the $\mathrm{K}$ ratio with changes in position (fig. $3 \mathrm{~b}$ ) suggests that the $\mathrm{K}$ ratio determined in one position does not reliably predict the corresponding value in another position, emphasizing the need for individual calibration in each position. Accurate determination of the $\mathrm{K}$ ratio is not only required for precise estimation of tidal volume by RIP, but it is also essential for appropriate detection of apnea/hypopnea and for assessment of the rib cage and abdominal contributions to tidal volume. Of note, the excellent accuracy of RIP in the estimation of respiratory timing variables does not seem to be critically dependent on calibration.

In a previous study using the same equipment [10] we obtained accurate estimates of tidal volumes during exercise in subjects and patients with advanced cardiac and pulmonary disease. In that study, calibration (QDC) and monitoring was performed in a single position, namely upright. The current study corroborates and extends these prior findings to a validation of RIP during variable positions at rest and during walking in unrestrained subjects and patients. Figures 4 and 5 as well as table 1 illustrate that the bias with position-dependent calibration was close to zero in subjects and patients in the various positions. In addition, the precision of tidal volume estimates by RIP was satisfactory as reflected in the limits of agreement with pneumotachographic values of $\pm 20 \%$ for breath-by-breath comparisons with the pneumotach- ograph including all positions in subjects and patients (table 1). An even greater precision (limits of agreement of $\pm 14 \%$ ) was achieved if tidal volumes were averaged over 10 breaths. These values compare favorably with those reported for a portable monitoring device based on magnetometers [19] and with our previous studies in healthy subjects using conventional RIP bands during maximal exercise (limits of agreement $\pm 18 \%$ ) [15] and in patients with advanced heart and lung disease using the LifeShirt (limits of agreement of $\pm 17 \%$ ) [10].

In conclusion, our study shows accurate estimation of tidal volumes by a lightweight, portable RIP device with sensors incorporated in a body garment using positionspecific calibration according to feedback from position sensors. The novel calibration technique greatly extends the application of portable RIP for monitoring the breathing pattern in unrestrained, ambulatory subjects and patients who repeatedly change body position such as during usual activities at home or in the workplace. Clinical applications of this unobtrusive technique may include ambulatory monitoring of patients with pulmonary, cardiovascular or neuromuscular disease during activities of daily living in order to better assess their ventilatory demands and limitations, to evaluate treatment effects and to correlate breathing patterns with symptoms and measures of energy expenditure obtained from accelerometers.

\section{Acknowledgment}

The study was supported by a grant from the Zurich Lung League. The RIP monitor (LifeShirt) was provided by VivoMetrics (Ventura, Calif., USA).

\section{References}

1 Sackner MA, Krieger BP: Noninvasive respiratory monitoring; in Scharf SM, Cassidy SS (eds): Heart-lung interactions in health and disease. New York, Marcel Dekker Inc., 1989, pp 663-805.

2 Thurnheer R, Xie X, Bloch KE: Accuracy of nasal cannula pressure recordings for assessment of ventilation during sleep. Am J Respir Crit Care Med 2001;164:1914-1919.

3 Kaplan V, Zhang JN, Russi EW, Bloch KE: Detection of inspiratory flow limitation during sleep by computer assisted respiratory inductive plethysmography. Eur Respir J 2000;15:570-578.
-4 Bloch KE, Li Y, Sackner MA, Russi EW: Breathing pattern during sleep disruptive snoring. Eur Respir J 1997;10:576-586.

5 Tobin MJ, Perez W, Guenther SM, Semmes BJ, Mador MJ, Allen MJ, Lodato RF, Dantzker DR: Pattern of breathing during successful and unsuccessful trials of weaning from mechanical ventilation. Am Rev Respir Dis 1986;134:1111-1118.

6 Bloch KE, Li Y, Zhang J, Bingisser R, Kaplan V, Weder W, Russi EW: Effect of surgical lung volume reduction on breathing patterns in severe pulmonary emphysema. Am J Respir Crit Care Med 1997;156:553-560.
7 Brack T, Thuer I, Clarenbach CF, Senn O, Noll G, Russi EW, Bloch KE: Daytime Cheyne-Stokes respiration in ambulatory patients with severe congestive heart failure is associated with increased mortality. Chest 2007;132:1463-1471.

8 Kohler M, Kriemler S, Wilhelm EM, Brunner-Larocca $\mathrm{H}$, Zehnder M, Bloch KE: Children at high altitude have less nocturnal periodic breathing than adults. Eur Respir J 2008;32:189-197.

-9 Ragnarsdottir M, Kristinsdottir EK: Breathing movements and breathing patterns among healthy men and women 20-69 years of age. Reference values. Respiration 2006; 73:48-54 
10 Clarenbach CF, Senn O, Brack T, Kohler M, Bloch KE: Monitoring of ventilation during exercise by a portable respiratory inductive plethysmograph. Chest 2005; 128:12821290.

11 Konno K, Mead J: Measurement of separate volume changes of ribcage and abdomen during breathing. J Appl Physiol 1966;22: 407-422.

12 Watson HL, Poole DA, Sackner MA: Accuracy of respiratory inductive plethysmographic measurement of cross-sectional area of models. J Appl Physiol 1988;65:306-308.
Zimmerman PV, Connellan SJ, Middleton HC, Tabona MV, Goldman MD, Pride N Postural changes in rib cage and abdominal volume-motion coefficients and their effect on the calibration of a respiratory inductance plethysmograph. Am Rev Respir Dis 1983;127:209-214.

14 Sackner MA, Watson H, Belsito AS, Feinerman D, Suarez M, Gonzalez G, Bizousky F, Krieger B: Calibration of respiratory inductive plethysmograph during natural breathing. J Appl Physiol 1989;66:410-420.

15 Bloch KE, Barandun J, Sackner MA: Effect of mouthpiece breathing on cardiorespiratory response to intense exercise. Am J Respir Crit Care Med 1995;151:1087-1092.

$\checkmark 16$ Bland MJ, Altman DG: Statistical methods for assessing agreement between two methods of clinical measurement. Lancet 1986; i:307-310.
17 Sackner JD, Nixon AJ, Davis B, Atkins N, Sackner MA: Non-invasive measurement of ventilation during exercise using a respiratory inductive plethysmograph. I. Am Rev Respir Dis 1980;122:867-871.

18 Chadha TS, Watson H, Birch S, Jenouri GA, Schneider AW, Cohn MA, Sackner MA: Validation of respiratory inductive plethysmography using different calibration procedures. Am Rev Respir Dis 1982;125:644-649.

19 McCool FD, Wang J, Ebi KL: Tidal volume and respiratory timing derived from a portable ventilation monitor. Chest 2002;122: 684-691. 